\title{
High-dose vs. low-dose vitamin D supplementation on phase angle values in older patients with prostate cancer on androgen deprivation therapy
}

\author{
Julia E. Inglis, Michelle C. Janelsins, Erika E. Ramsdale, Richard F. Dunne, Gilberto \\ Lopez, Eva Culakova, Amber S. Kleckner, Po-Ju Lin, lan R. Kleckner, Kah Poh Loh, \\ Luke J. Peppone
}

Introduction: Vitamin D insufficiency may be a factor in frailty in cancer and in older adults. Low vitamin $D$ is associated with decreased lean mass, physical function and quality of life. Phase angle, calculated from bioelectrical impedance analysis (BIA), is a an indicator or muscle and tissue integrity as well as frailty.

Objective: To investigate if high dose vitamin D supplementation is associated with phase angle values as a potential frailty indicator.

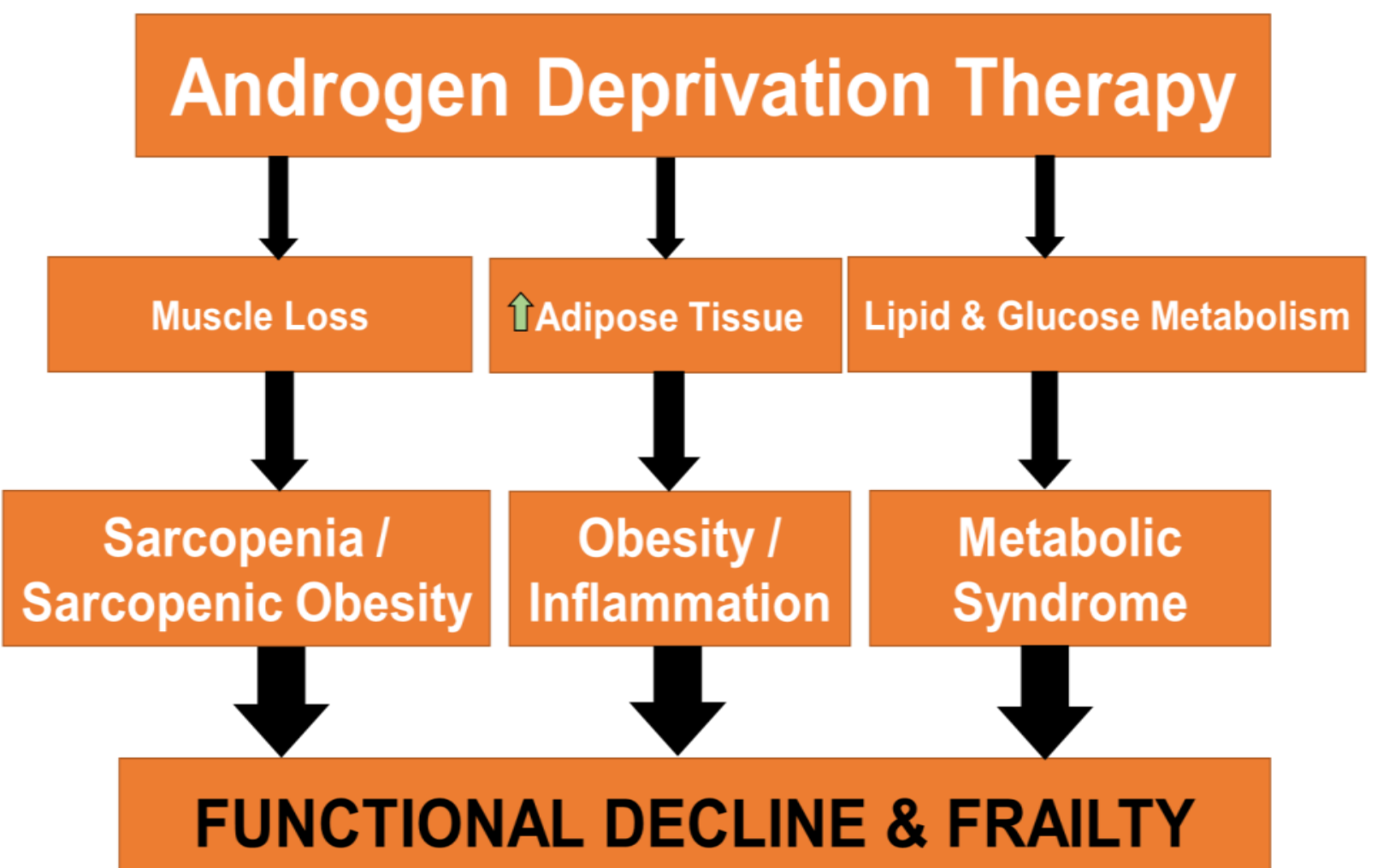

Methods: A secondary analysis of patients with prostate cancer $(\mathrm{N}=59$, age $67.6 \pm 5.4)$ and vitamin $\mathrm{D}$ insufficiency $(<32 \mathrm{ng} / \mathrm{ml})$ randomized to 1 of 2 intervention arms for 24 weeks:

Arm 1: Low-dose: RDA for vitamin D ( $n=30,600$ IU/daily plus placebo weekly)

Arm 2: High-dose vitamin D ( $n=29,600$ IU/daily plus 50,000 IU/weekly).

Phase Angle Values Between Groups: $12 \& 24$ Weeks

6

5.9

5.8

5.7

5.6

5.5

5.4

5.3

5.2

5.1

5

\section{Vitamin D Levels Between Arms by Assessment Point}

Vitamin D Levels $\mathrm{ng} / \mathrm{mL}$

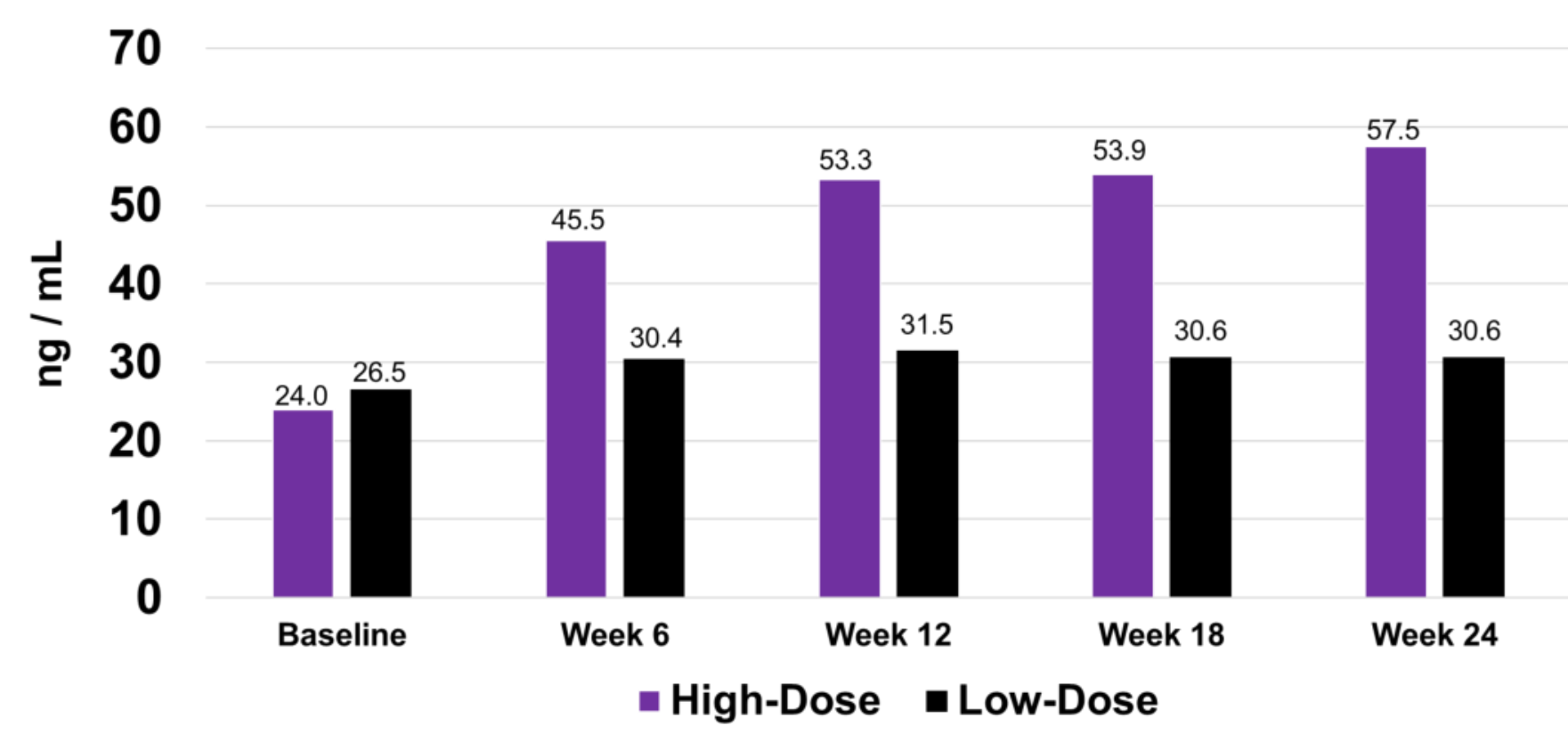

Methods: BIA was conducted at baseline, 12 and 24 weeks.

- Phase angle calculations (BIA): arctan (reactance/resistance) $\times(180 \% \pi)$.

- A phase angle value $<5.7^{\circ}$ is a validated cutoff for frailty in older men.

- Multivariate regression evaluated differences in phase angle values between arms.

Results:

- High-dose vitamin $D$ group had wider phase angle values at 12 weeks (5.81ํ vs. $\left.5.32^{\circ} ; p=0.017\right)$ and 24 weeks (5.89 vs. $\left.5.40^{\circ} ; p=0.007\right)$ than low-dose group.

- Low-dose group had phase angle values $<5.7^{\circ}$ over the course of the study, whereas the high-dose vitamin $D$ group maintained values $>5.8^{\circ}$.

Conclusion: The high-dose vitamin $D$ group maintained wider phase angle values over the course of 24 weeks, suggesting less frailty, while phase angle values for the low-dose group declined from baseline. The BIA is a low-cost, portable tool for assessing phase angle in cancer patients.

Changes in Phase Angle from Baseline: 12 Weeks to 24 Weeks 0.3

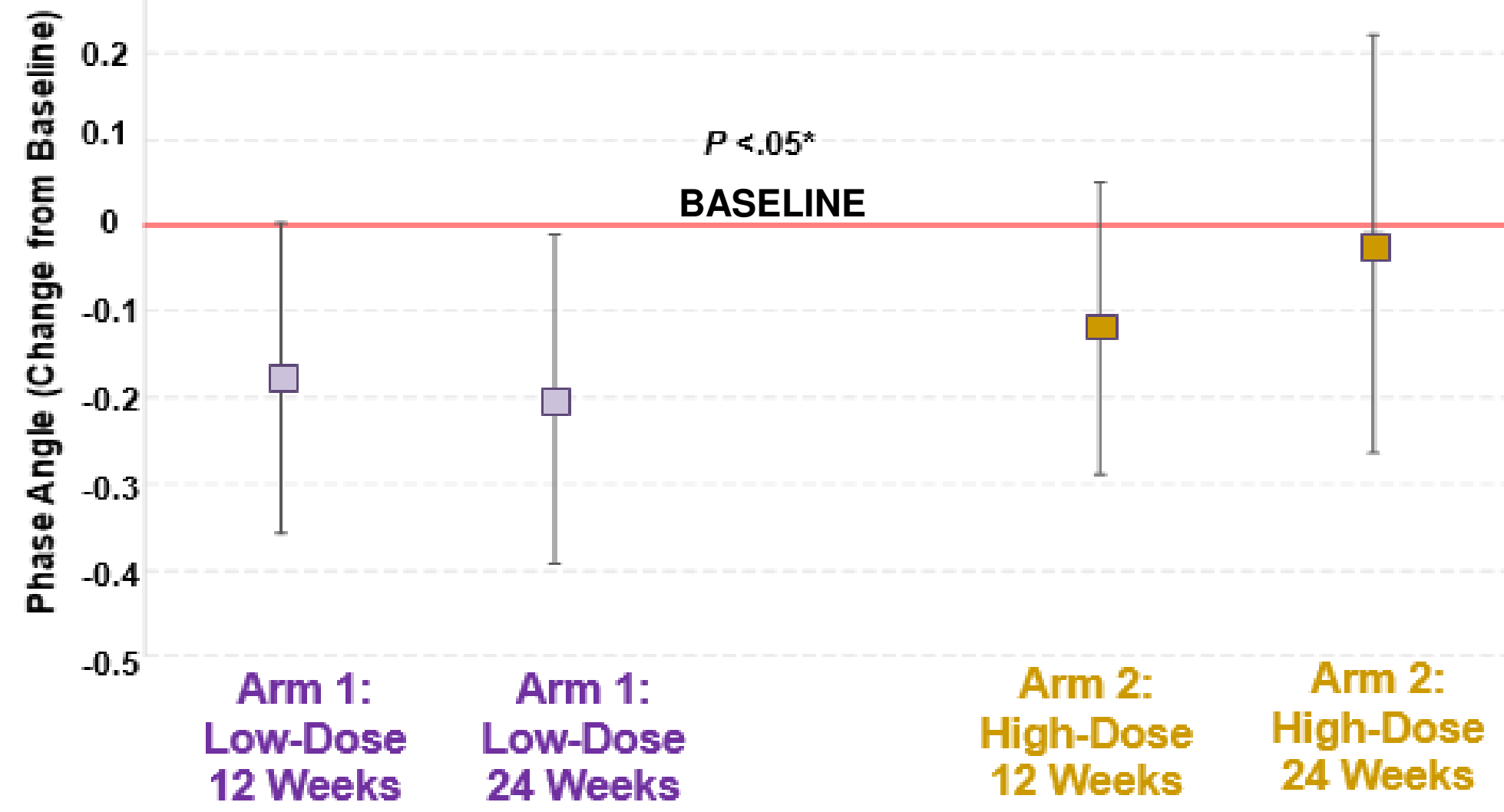

*Differences in changes in phase angle values significantly different between low-dose and high dose groups, at both 12 weeks and 24 weeks. 